\title{
ESSAIS D'UTILISATION DE L'INFRA RED MILK ANALYZER
}

\author{
par \\ J. ADDA (2), E. BLANC-PATIN (1), R. JEUNET (1) \\ R. GRAPPIN (1), G. MOCQUOT (2), B. POUSARDIEU (3) \\ et G. RICORDEAU (3)
}

\section{Introduction}

Le présent travail se rapporte à l'analyse des principaux composants du lait (matière grasse, matière azotée, lactose) par spectrophotométrie infra-rouge. Le travail initial est dû à Goulden $(1958,1961)$ qui a conçu, puis réalisé, un spectrophotomètre infra-rouge (Infra Red Milk Analyzer, en abrégé IRMA) permettant de doser, en une minute environ, la matière grasse, la matière azotée et le lactose sur un échantillon de lait de $40 \mathrm{ml}$, sans extraction ni minéralisation.

L'appareil dans sa première version, (MK I), a déjà été expérimenté en Grande-Bretagne, au Milk Marketing Board, et surtout au Canada par le professeur Biggs (4) de l'université de Guelph (Ontario) dont les travaux ont été déterminants dans la mise au point d'une seconde version, (MK II), très améliorée de l'appareil.

Nous avons pu, grâce à l'obligeance du constructeur (5), disposer pendant plusieurs mois d'un appareil de type MK I à la Station Expérimentale Laitière de Poligny et le soumettre à une série de tests dont les résultats ont déjà été diffusés dans un rapport interne.

D'autre part, la description de l'appareil a déjà fait elle-même l'objet de plusieurs publications (Goulden, Shields et Haswell, 1964 ; Alais, 1965; Biggs, 1967) mais il semble néanmoins utile de rappeler brièvement le principe de la méthode et son application.

Auteurs cités par ordre alphabétique :

(1) Station expérimentale laitière, Poligny (Jura).

(2) Station centrale de recherches laitières et de technologie des produits animaux, C.N.R.Z., Jouy-en-Josas (Yvelines).

(3) Station centrale de génétique animale, C.N.R.Z., Jouy-en-Josas (Yvelines).

(4) Que nous tenons à remercier ici pour avoir accueilli deux d'entre nous dans son laboratoire.

(5) Sir H. Grubb-Parson Ltd. Newcastle upon Tyne. Grande-Bretagne. 


\section{Principe de la méthode}

L'absorption quantitative du rayonnement infra-rouge, à 5,73 microns par la liaison ester des triglycérides, à 6,46 microns par la liaison amide II des protéines et à 9,60 microns par les groupes hydroxyles du lactose, permet de doser ces composants dans le lait. Comme il y a une absorption considérable d'énergie infra-rouge par l'eau dans la zone de longueur d'onde utilisée, une absorption constante est soustraite en utilisant une cellule de référence pleine d'eau.

\section{Réalisation et fonctionnement de l'appareil}

L'énergie émise parr une source infra-rouge (filament de Nernst) est divisée en deux faisceaux dont l'un passe au travers d'une cellule de référence contenant de l'eau tandis que l'autre passe au travers d'une cellule contenant le lait. Ces deux cellules, dont les parois sont en fluorure de calcium, ont une épaisseur utile de 40 microns environ. Les deux faisceaux suivent ensuite alternativement le même chemin optique grâce à un miroir oscillant et pénètrent dans un système monochromatique, constitué d'un réseau et d'un prisme qui sélectionne les longueurs d'ondes correspondant au maximum d'absorption de chacun des composants à analyser. Le faisceau tombe ensuite sur le détecteur qui est un thermocouple ultrasensible. Le lait et l'eau absorbent des quantités d'énergie différentes; il en résulte que l'énergie transportée par le faisceau recombiné varie à chaque alternance et qu'au niveau du détecteur cela donne naissance à un courant alternatif qui est amplifié puis redressé. Ce courant fait mouvoir un petit moteur sur l'axe duquel est fixé une came à contour logarithmique. Le rotation de cette came introduit un peigne atténuateur dans la faisceau lumineux qui passe au travers de la cellule de référence, réduisant ainsi l'énergie transmise. Le mouvement de ce peigne se poursuit tant qu'il n'y a pas équilibre entre les deux faisceaux et se traduit par un signal qui est amplifié et enregistré.

L'importance de la correction effectuée par l'introduction du peigne dans le faisceau est proportionnelle à la concentration du lait en éléments à doser.

En fait, conformément à la loi de Lambert-Beer, la relation entre concentration et déplacement du peigne est logarithmique mais le tracé logarithmique de la came rend le résultat linéaire.

\section{Appareillage et mise en route}

Après un chauffage préliminaire, qui amène le lait à $38^{\circ} \mathrm{C}$, l'échantillon de $40 \mathrm{ml}$ de lait environ est soigneusement mélangé par retournement, homogénéisé de façon que le diamètre des globules gras ne soit pas supérieur à deux microns. Le lait aspiré 
par pompage passe dans un échangeur de température qui l'amène à $1^{0} \mathrm{C}$ près à une température bien définie et traverse ensuite la cellule de mesure. La plus grande partie de l'échantillon ne sert qu'à chasser l'échantillon précédent et à rincer la cellule; ceci est réalisé après environ 25 secondes de pompage.

Il faut prévoir 2 opérateurs pour ce type d'appareil : l'un réchauffe et homogénéise les échantillons, l'autre effectue les dosages.

Dès que le pompage a cessé, on appuie sur le bouton qui permet, par l'intermédiaire d'une came, de sélectionner successivement les longueurs d'ondes correspondant au maximum d'absorption des différents constituants et le résultat, exprimé en grammes p. 100 grammes de lait, est lu directement sur un cadran numérique ou mesuré par la déviation de la plume d'un enregistreur.

\section{Etalonnage de l'appareil}

L'étalonnage initial est réalisé pour chacun des trois composants par l'analyse d'un nombre important d'échantillons, de composition aussi différente que possible, par spectrophotométrie infrarouge d'une part et par une méthode chimique d'autre part. Lorsque l'étalonnage est réalisé, les deux séries de résultats doivent être identiques. On peut alors représenter cette relation par une droite qui passe par l'origine et qui a une pente égale à 1 . En raison d'interférences on obtient généralement des résultats IRMA inférieurs à ceux fournis par les dosages chimiques. Un dispositif de correction ("ranging unit») qui permet soit d'ajouter au signal fourni par le détecteur une différence de potentiel, soit de multiplier le signal par une constante, donne le moyen d'amener les deux séries de résultats à des valeurs identiques.

Il ne saurait être question de vérifier l'étalonnage de l'appareil par des analyses ehimiques quotidiennes. Aussi l'appareil est-il muni de deux filtres qui, interposés dans le faisceau optique, donnent une absorption constante à une longueur d'onde donnée. Les valeurs de ces absorptions sont déterminées aux différentes longueurs d'onde après étalonnage initial de l'appareil. On s'assure quotidiennement que cet étalonnage n'a pas varié en vérifiant que les valeurs fournies par les filtres sont toujours les mêmes. Si l'on n'obtient pas la valeur désirée, ce qui indique une légère variation par rapport à l'étalonnage initial, le dispositif de contrôle permet d'apporter les ajustements nécessaires. Ces filtres, très utiles pour vérifier la stabilité de l'étalonnage au cours par exemple d'une journée de travail, ne peuvent suffire à garantir cette stabilité sur une longue période, ne serait-ce qu'en raison du risque d'altération des filtres. En outre.les filtres ne permettent pas de transférer l'étalonnage d'un appareil à un autre car ils ne peuvent pas être rigoureusement identiques sur deux appareils différents. 
Pour obvier à cette difficulté, on utilise des solutions de corps chimiquement purs. Ces solutions, qui sont préparées avec la précision que permet d'obtenir la chimie analytique, absorbent respectivement aux mêmes longueurs d'ondes que la matière grasse, la matière azotée et le lactose. Leur absorption a été déterminée à deux concentrations différentes sur un appareil rigaureusement étalonné par analyse d'un grand nombre d'échantillons de lait. Il devient dès lors possible, si l'on est disposé à accepter les valeurs fournies par un laboratoire central, d'étalonner de façon identique de très nombreux "IRMA " en ajustant les dispositifs de contrôle pour retrouver l'absorption que doit avoir chaque solution étalon.

Les valeurs particulières que fournissent les filtres de chaque appareil, ainsi étalonné, seront ensuite utilisées pour maintenir l'étalonnage.

Ceci permet de substituer, à une multitude de critères de précision locaux, un critère unique et un moyen de standardisation

TABLEAU I

\begin{tabular}{|c|c|c|}
\hline Nature du contrôle & Fréquence & Par \\
\hline $\begin{array}{l}\text { Température de l'échangeur detem- } \\
\text { pérature et de l'instrument } \\
\text { Lecture de valeur du filtre } \\
\text { Equilibre électronique } \\
\text { Zéro optique } \\
\text { Ligne de base } \\
\text { Valeurs de solutions chimiques } \\
\text { Débit à travers la cellule } \\
\text { Efficacité de l'homogénéisateur } \\
\text { Nettoyage du circuit lait } \\
\text { Nettoyage de la cellule de référence } \\
\text { Nettoyage de l'homogénéisateur } \\
\text { Démontage de la tête de l'homogé- } \\
\text { néiseur (changement des joints) } \\
\text { Inspection du Nernst et de son ali- } \\
\text { mentation } \\
\text { Vérification de la stabilité des solu- } \\
\text { tions étalons } \\
\text { Changement du Nernst } \\
\text { Changement de cellule } \\
\text { Démontage de cellule } \\
\text { Réalignement optique } \\
\text { Réajustement de la longueur d'onde } \\
\text { Réétalonnage de l'appareil }\end{array}$ & $\begin{array}{l}\text { Toutes les heures } \\
\text { Toutes les } 2 \text { heures } \\
\text { Le plus souvent possible } \\
\text { Quotidien } \\
\text { Quotidien } \\
\text { Quotidien } \\
\text { Tous les dix échantillons } \\
\text { Tous les dix échantillons } \\
\text { Biquotidien } \\
\text { Quotidien } \\
\text { Quotidien } \\
\text { Bi-mensuel } \\
\text { Hebdomadaire } \\
\text { Bi-mensuel } \\
\text { Selon les besoins } \\
\text { Selon les besoins } \\
\text { Selon les besoins } \\
\text { Selon les besoins } \\
\text { Mensuel } \\
\text { En cas de changement de cellule }\end{array}$ & $\begin{array}{l}\mathrm{T} \\
\mathrm{T} \\
\mathrm{T} \\
\mathrm{T} \\
\mathrm{T} \\
\mathrm{T} \\
\mathrm{T} \\
\mathrm{T} \\
\mathrm{T} \\
\mathrm{T} \\
\mathrm{T} \\
\mathrm{TS}\end{array}$ \\
\hline
\end{tabular}

T : Technicien.

TS : Technicien supérieur. 
des appareils des laboratoires agréés, garantissant une unité de résultats quel que soit le laboratoire qui a effectué l'analyse. Il est évident que la précision de l'étalonnage initial dépend de la fidélité et de la précision des méthodes chimiques de référence. Ensuite toute la valeur des résultats que l'on peut obtenir avec IRMA dépend de l'habileté avec laquelle l'opérateur maintient, jour après jour, l'étalonnage de l'appareil au moyen, à la fois, des filtres et des solutions chimiques de référence.

En plus de ceux destinés à maintenir l'étalonnage, l'utilisateur doit effectuer un certain nombre de contrôles dont nous indiquons la fréquence dans le tableau I mais ces contrôles ne demandent que quelques minutes par jour.

L'étalonnage correct de l'appareil requiert une certaine maîtrise des opérations et il semble évident que les déboires initiaux de tous ceux, (y compris les auteurs du présent travail), qui se sont servis de l'IRMA proviennent en grande partie d'un manque d'habitude. L'étalonnage initial étant réalisé par l'installateur, on est amené par la suite à réaliser de légers ajustements quotidiens; mais si ces légères corrections se révèlent insuffisantes il faut chercher une autre cause au mauvais fonctionnement (cellule encrassée, mauvaise homogénéisation du lait, fluctuations de la température, anomalies de fonctionnement des circuits électroniques).

L'utilisation de solutions de référence préparées à partir de produits chimiques purs ne présente pas de difficultés particulières pour l'étalonnage aux longueurs d'onde où absorbent matière azotée et lactose puisqu'on utilise alors du propionate de calcium et du lactose, produits stables. L'utilisation de butyrolactone pour l'étalonnage à la longueur d'onde où absorbe la matière grasse nécessite quelques précautions puisque d'une part la butyrolactone est très hygroscopique et que d'autre part ses solutions ne sont pas stables.

\section{Reproductibilité et précision de la méthode}

Pour déterminer la valeur de l'appareil IRMA, nous avons effectué deux séries de tests : les uns concernant la reproductibilité des mesures, les autres leur précision. Dans chaque série, des comparaisons ont été faites avec les méthodes rapides d'analyse : Gerber (Terroine, 1951), Noir amido (Posthumus, 1959), ou avec les méthodes chimiques de référence : Röse Gottlieb (Norme Fil 1 : 1955), Kjeldahl (Rowland, 1938) et Bertrand (Terroine, 1951).

La plus grande partie de nos essais a porté sur les dosages de matière grasse et de matière azotée qui sont les constituants du lait les plus importants. Un certain nombre de dosages de lactose, dont les résultats sont présentés plus loin, ont également été effectués. 


\section{A. - Analyse de la matière grasse et de la matière azotée}

1) Protocole expérimental.

Un premier essai a porté sur 71 échantillons de laits de vache individuels (animaux de race Montbéliarde) prélevés immédiatement après la traite et conservés au froid.

Chaque échantillon a été analysé dans un délai de 2 à 3 jours à la fois par IRMA et par voie chimique.

- Analyse par IRMA : chacun des échantillons a été soumis à 7 analyses successives qui ont fourni 7 résultats de matière grasse et 7 résultats de matière azotée.

- Analyses chimiques : la matière azotée a été dosée par la méthode colorimétrique au Noir amido ( 7 analyses successives sur chacun des 71 échantillons de lait) et par la méthode Kjeldahl ( 2 analyses sur chacun des 71 échantillons).

TABLEAU II

ANALYSE DE LA VARIANCE DES 7 RÉPÉtitions EN FONCTION DE LA MÉTHODE DE DOSAGE

\begin{tabular}{|c|c|c|c|}
\hline Source de variation & $\begin{array}{l}\text { Nombre de } \\
\text { degré de liberté }\end{array}$ & $\begin{array}{l}\text { Somme des } \\
\text { carrés }\end{array}$ & $\begin{array}{l}\text { Estimation de } \\
\text { la variance }\end{array}$ \\
\hline $\begin{array}{l}\text { Matière azotée IRMA } \\
\text { entre laits : } \\
\text { intra-lait : }\end{array}$ & $\begin{array}{r}70 \\
426\end{array}$ & $\begin{array}{r}6316,99 \\
10,24\end{array}$ & $\begin{array}{c}12,8884 \\
0,02404\end{array}$ \\
\hline $\begin{array}{l}\text { Noir A mido } \\
\text { entre laits : } \\
\text { intra-lait : }\end{array}$ & $\begin{array}{r}70 \\
426\end{array}$ & $\begin{array}{r}619,71 \\
1,37\end{array}$ & $\begin{array}{c}14,1214 \\
0,00323\end{array}$ \\
\hline $\begin{array}{l}\text { Matière grasse IRMA } \\
\text { entre laits : } \\
\text { intra-lait : }\end{array}$ & $\begin{array}{r}70 \\
426\end{array}$ & $\begin{array}{r}379,97 \\
17,21\end{array}$ & $\begin{array}{l}76,2799 \\
0,04040\end{array}$ \\
\hline $\begin{array}{l}\text { Gerber } \\
\text { entre laits : } \\
\text { intra-lait : }\end{array}$ & $\begin{array}{r}70 \\
426\end{array}$ & $\begin{array}{r}37 \quad 141,33 \\
22,01\end{array}$ & $\begin{array}{l}75,7913 \\
0,05167\end{array}$ \\
\hline
\end{tabular}

La matière grasse a été dosée par la méthode Gerber (à raison de 7 répétitions sur chacun des 71 échantillons de lait) et par la méthode Röse Gottlieb ( 2 répétitions sur 30 échantillons de lait; pour les 41 autres échantillons de lait on a pris comme valeur de référence la moyenne corrigée (1) de 7 analyses Gerber).

(1) On a constaté un écart systématique de $-0,30 \mathrm{~g} / \mathrm{kg}$ entre la moyenne des 7 analyses Gerber et la moyenne des 2 analyses Rose Gottlieb. 
D'autres essais, souvent moins complets que celui-ci, dont le but était de préciser certains points particuliers, seront mentionnés au cours des paragraphes suivants.

2) Résultats.

a) Reproductibilité des différentes méthodes :

Pour les 4 méthodes d'analyse suivantes : Matière grasse IRMA, matière azotée IRMA, matière grasse-Gerber, matière azotée Noir amido, nous avons effectué une analyse de variance à une voie afin d'estimer la variabilité des résultats pour chacune des 4 méthodes (Tableau II).

En prenant comme base l'écart type résiduel (qui caractérise la reproductibilité moyenne), les différentes méthodes se classent de la façon suivante :

$$
\begin{aligned}
& \text { Matière azotée } \begin{cases}\text { Noir amido } & ; \sigma=0,06 \mathrm{~g} / \mathrm{kg} \\
\text { IRMA } & : \sigma=0,16 \mathrm{~g} / \mathrm{kg}\end{cases} \\
& \text { Matière grasse } \begin{cases}\text { IRMA } & ; \sigma=0,20 \mathrm{~g} / \mathrm{kg} \\
\text { Gerber } & ; \sigma=0,23 \mathrm{~g} / \mathrm{kg}\end{cases}
\end{aligned}
$$

En ce qui concerne l'appareil IRMA, cela signifie que l'erreur " intra lait " à 95 p. 100 est égale à $0,32 \mathrm{~g} / \mathrm{kg}$ pour la matière azotée et à $0,40 \mathrm{~g} / \mathrm{kg}$ pour la matière grasse.

\section{TABLEAU III}

ANALYSE DE LA VARIANCE DE MESURES IRMA

\begin{tabular}{|c|c|c|c|c|}
\hline Source de variation & $\begin{array}{l}\text { Nombre de } \\
\text { degrés de } \\
\text { liberté }\end{array}$ & $\begin{array}{l}\text { Somme } \\
\text { des } \\
\text { carrés }\end{array}$ & $\begin{array}{c}\text { Estimation } \\
\text { de la } \\
\text { variance }\end{array}$ & $F$ \\
\hline $\begin{array}{l}\qquad \text { Matière grasse } \\
\text { Totale........... } \\
\text { Effet date .... } \\
\text { Effet lait ..... } \\
\text { Variation intra-lait } \\
\text { (date + résiduelle) } \\
\text { Résiduelle ...... }\end{array}$ & $\begin{array}{r}143 \\
11 \\
11 \\
132 \\
121\end{array}$ & $\begin{array}{c}7281,67 \\
13,67 \\
7257,53 \\
\\
24,14 \\
10,47\end{array}$ & $\begin{array}{c}1,242 \\
659,77 \\
0,1829 \\
0,0865\end{array}$ & $+t$ \\
\hline $\begin{array}{l}\quad \text { Matière azotée } \\
\text { Totale.......... } \\
\text { Effet date ..... } \\
\text { Effet lait ....... } \\
\text { Variation intra-lait } \\
\text { (date + résiduelle) } \\
\text { Résiduelle ...... }\end{array}$ & $\begin{array}{r}132 \\
10 \\
11 \\
121 \\
111\end{array}$ & $\begin{array}{c}820,44 \\
3,10 \\
809,22 \\
11,22 \\
8,12\end{array}$ & $\begin{array}{c}0,310 \\
73,565 \\
\\
0,0927 \\
0,0731\end{array}$ & $+t$ \\
\hline
\end{tabular}
RÉPÉtétes 12 FOIS SUR UNE PÉRIOdE DE 15 JOURS 
Avec la méthode au Noir amido, la variance résiduelle est significativement plus petite (test F) qu'avec le dosage de la matière azotée par IRMA. Par contre les variances résiduelles des teneurs en matière grasse ne sont pas significativement différentes, que l'on. travaille avec IRMA ou avec la méthode Gerber.

Il faut bien souligner que ces valeurs représentent la reproductibilité de l'appareil sur une courte période, pendant laquelle les différents éléments qui peuvent modifier la réponse de l'appareil (température, homogénéisation, étalonnage, etc.) n'ont pas varié. En effet, dans un essai portant sur 12 échantillons conservés au froid avec le conservateur fourni par le constructeur de l'appareil et analysés chaque jour pendant une période de 2 semaines, une augmentation importante de l'erreur de mesure a pu être mise en évidence : l'écart type résiduel IRMA est de $0,30 \mathrm{~g} / \mathrm{kg}$ pour le dosage de la matière azotée et de $0,43 \mathrm{~g} / \mathrm{kg}$ pour le dosage de la matière grasse. Ces valeurs sont supérieures à celles obtenues dans le premier essai (respectivement 0,16 et 0,20 ). L'analyse de la variance (Tableau III) met en évidence un "effet date " très significatif qui provient sans doute d'une variation dans l'étalonnage (en supposant bien entendu que la composition des laits n'a pas varié au cours de la conservation).

La reproductibilité des résultats sur une longue période sera d'autant meilleure que l'utilisateur saura mieux faire preuve d'habileté et de compétence pour maintenir l'étalonnage parfaitement constant.

\section{TABLEAU IV}

ECART-TYPE ( $\mathrm{g}$-kg) DES DIFFÉRENCES ENTRE LA MÉTHODE RAPIDE ET LA MÉTHODE DE RÉFÉRENCE (71 LAITS)

\begin{tabular}{|c|c|c|}
\hline Méthode rapide &,$\quad \sigma$ & Méthode de référence \\
\hline Noir amido & 0,40 & Kjeldahl \\
\hline Gerber & 0,46 & Röse Gottlieb \\
\hline Matière azotée IRMA & 0,62 & Kjeldahl \\
\hline Matière grasse IRMA & 1,02 & Röse Gottlieb \\
\hline
\end{tabular}

b) Précision de la méthode :

Les méthodes de référence utilisées pour tester la précision de l'appareil IRMA sont les méthodes Kjeldahl pour la matière azotée et Röse Gottlieb pour la matière grasse. Cependant, dans certains essais portant sur un nombre élevé d'échantillons, nous 
avons substitué à ces méthodes de référence les méthodes Gerber et Noir amido parce qu'elles sont plus rapides.

Dans le cas où l'appareil est juste, c'est-à-dire correctement étalonné, la précision est estimée en calculant l'écart-type des différences observées par rapport à la méthode de référence.

Lors de la première série d'essais portant sur 71 échantillons de lait, les résultats fournis par l'IRMA, la méthode colorimétrique au Noir amido et la méthode Gerber ont été comparés à ceux obtenus à l'aide des méthodes de référence, Röse Gottlieb et Kjeldahl. L'écart-type des différences observées entre la méthode rapide et la méthode de référence donne une estimation de la précition de la méthode rapide (Tableau IV).

D'après ces premiers résultats, la méthode IRMA apparaît moins précise que les autres méthodes habituelles : Noir amido et surtout Gerber.

En comparant la méthode IRMA aux méthodes Gerber et Noir amido, l'importance des écarts est du même ordre que celle observée lorsqu'on prend en considération les méthodes de référence : l'écart-type des différences entre matière azotée IRMA et matière azotée Noir amido est de $0,593 \mathrm{~g} / \mathrm{kg}$; il est de $0,973 \mathrm{~g} / \mathrm{kg}$ entre matière grasse IRMA et matière grasse Gerber.

TABLEAU V

VARIATIONS DE L'ÉCHANTILLONNAGE DE L'APPAREIL EN FONCTION DE L'ÉCHANTILLONNAGE DE LAITS ANALXSÉG

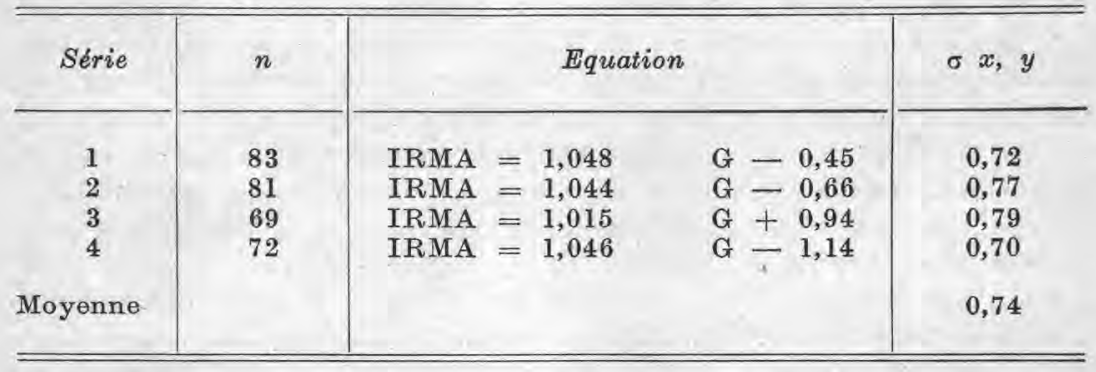

$\mathrm{Au}$ cours d'essais ultérieurs portant uniquement sur la détermination du taux butyreux, les résultats de la méthode Gerber ont été comparés à ceux obtenus par une lecture unique avec l'IRMA. On a analysé 305 échantillons de laits individuels prélevés par des contrôleurs laitiers dans des flacons contenant une pastille du conservateur fourni par le construeteur de l'appareil. Les échantillons ont été analysés en 4 séries distinctes au cours d'une période de 10 jours. Pour chaque série on a calculé l'équation de la droite de régression et l'écart-type lié qui permet d'estimer la précision de la méthode (Tableau V). La moyenne des écarts-type est de $0,74 \mathrm{~g} / \mathrm{kg}$. Ce résultat est très proche de celui trouvé par Biggs 
(1967) qui a comparé les résultats obtenus par IRMA à ceux obtenus par la méthode Babcock et cette valeur semble bien correspondre aux performances que l'on peut attendre de l'appareil en ce qui concerne la détermination du taux butyreux.

Cette amélioration de $0,23 \mathrm{~g}(0,97-0,74)$ de la précision des dosages de matière grasse par rapport aux premiers essais peut provenir d'une meilleure connaissance et d'une meilleure utilisation de l'appareil.

De ces essais on peut tirer la conclusion suivante : si les équations du tableau $V$ sont différentes entre elles, cela peut provenir de variations dans l'étalonnage non décelées lors des contrôles (filtre étalon et solution standard); mais cela peut provenir aussi de de l'échantillonnage de laits analysés. Cet exemple montre bien que, pour réaliser l'étalonnage initial de l'appareil à partir des analyses chimiques, il faut doser un nombre très important de laits afin d'éliminer, justement, les erreurs d'échantillonnage.

\section{B. - Dosage du lactose}

On a prélevé 44 échantillons de laits individuels sur lesquels la matière grasse, la matière azotée et le lactose ont été analysés par IRMA. Parallèlement le lactose a été dosé par la méthode Bertrand.

Le dosage du lactose par IRMA dépend en partie de la teneur du lait en matière grasse et en matière azotée, l'un de ces constituants provoquant une erreur par défaut et l'autre une erreur par excès. Le constructeur de l'IRMA a admis que ces deux erreurs se compensaient puisqu'il existe en général une corrélation positive entre taux butyreux et teneur en matière azotée; il n'est donc pas prévu sur l'appareil de dispositif pour corriger automatiquement le résultat obtenu pour le lactose, en fonction des teneurs des autres constituants. Dans cet essai, la précision du dosage du lactose avec IRMA est égale à $0,78 \mathrm{~g} / \mathrm{kg}$ (écart-type lié des différences par rapport à la méthode de référence). Les échantillons analysés provenaient de laits d'une seule traite dont la composition en graisse et matière azotée variait dans une gamme assez large (de 26 à $70 \mathrm{~g} / \mathrm{kg}$ pour le taux butyreux et de 24 à $46 \mathrm{~g} / \mathrm{kg}$ pour le taux de matière azotée) : ceci explique sans doute pourquoi nos résultats sont légèrement moins bons que ceux obtenus par Goulden (1964) et par Biggs (1967). 